\title{
New Results on Oscillation of even Order Neutral Differential Equations with Deviating Arguments
}

\author{
Lianzhong Li ${ }^{1}$, Fanwei Meng ${ }^{2}$ \\ ${ }^{1}$ School of Mathematical and System Sciences, Taishan College, Tai'an, China \\ ${ }^{2}$ School of Mathematical Sciences, Qufu Normal University, Qufu, China \\ E-mail: llz3497@163.com, fwmeng@qfnu.edu.cn \\ Received January 19, 2011; revised March 15, 2011; accepted March 20, 2011
}

\begin{abstract}
In this paper, we point out some small mistakes in [6] and revise them, we obtain some new oscillation results for certain even order neutral differential equations with deviating arguments. Our results extend and improve many known oscillation criteria because the article just generalizes Meng and Xu's results.
\end{abstract}

Keywords: Oscillation, Neutral Differential Equation, Deviating Argument

\section{Introduction}

Oscillation of some even order differential equations have been studied by many authors. For instance, see [1-7] and the references therein. We deal with the oscillatory behavior of the even order neutral differential equations with deviating arguments of the form

$$
\begin{aligned}
& {\left[x(t)+\sum_{i=1}^{m} p_{i}(t) x\left(\tau_{i}(t)\right)\right]^{(n)}} \\
& +\sum_{j=1}^{l} q_{j}(t) f_{j}\left(x\left(\sigma_{j}(t)\right)\right) \\
& =0, \quad t \geq t_{0}
\end{aligned}
$$

where $n \geq 2$ is even, throughout this paper, it is assumed that:

$\left(\mathrm{A}_{1}\right) \quad p_{i}, q_{j} \in C\left(\left[t_{0}, \infty\right), R^{+}\right), f_{j} \in C(R, R), u f_{j}(u)>0$ for $u \neq 0$ and $f_{j}(u)$ is non-decreasing on $R$, $i=1,2, \cdots, m, \quad j=1,2, \cdots, l$;

$\left(\mathrm{A}_{2}\right) \tau_{i} \in C\left(\left[t_{0}, \infty\right), R^{+}\right), \quad t_{i}(t) \leq t$ and $\lim _{t \rightarrow \infty} \tau_{i}(t)=\infty$, $i=1,2, \cdots, m$;

$\left(\mathrm{A}_{3}\right) \sigma_{j} \in C^{1}\left([t, \infty), R^{+}\right), \quad \sigma_{j}(t) \leq t, \quad \lim _{t \rightarrow \infty} \sigma_{j}(t)=\infty$ and $\sigma_{j}^{\prime}(t) \geq 0, j=1,2, \cdots, l$;

$\left(\mathrm{A}_{4}\right)$ There exists a constant $\mathrm{M}>0$ such that $f_{j}(x) \operatorname{sgn}(x) \geq \mathrm{M}|x|$ for $x \neq 0, j=1,2, \cdots, l$;

(As) $\sum_{i=1}^{m} p_{i}(t) \leq p, p \in(0,1)$ and there exists a function $q(t) \in C\left(\left[t_{0}, \infty\right), R^{+}\right)$such that, $q(t) \leq \min \left\{q_{j}(t)\right.$ : $j=1,2, \cdots, l\}$.

By a solution of Equation (1) we mean a function $x(t)$ which has the property that $x(t)+\sum_{i=1}^{m} p_{i}(t) x\left(\tau_{i}(t)\right)$ $\in C^{n}\left(\left[t_{x}, \infty\right), R\right)$ for some $t_{x} \geq t_{0}$ and satisfies Equation (1) on $\left[t_{x}, \infty\right)$. We restrict our attention to those solutions $x(t)$ of Equation (1) which exist on some half-line $\left[t_{x}, \infty\right)$ with $\sup \{|x(t)|: t \geq T\} \neq 0$ for any $T \geq t_{x}$. A nontrivial solution of Equation (1) is called oscillatory if it has arbitrarily large zeros, otherwise it is said to be nonoscillatory. Equation (1) is said to be oscillatory if all of it's nontrivial solutions are oscillatory.

Recently, Meng and Xu [6] studied Equation (1) and obtained some sufficient conditions for oscillation of the Equation (1), we list the main results of [6] as follows.

Following Philos [5], we say that a function $H=H(t, s)$ belongs to a function class $W$, denotes by $H \in W$, if $H \in C\left(D, R^{+}\right)$, where $D=\left\{(t, s): t \geq s \geq t_{0}\right\}$, which satisfies: $\left(\mathrm{H}_{1}\right) H(t, t)=0$ and $H(t, s)>0$ for $t_{0}<s<t<\infty ;\left(\mathrm{H}_{2}\right) H$ has a continuous non-positive partial derivative $\frac{\partial H}{\partial S}$ satisfying the condition:

$$
\frac{\partial H(t, s)}{\partial S}=h(t, s)-H(t, s) \frac{k^{\prime}(s)}{k(s)}
$$

for some $h \in L_{\text {loc }}(D, R), \quad k \in C^{1}\left(\left[t_{0}, \infty\right),(0, \infty)\right)$ is a non-decreasing function.

Theorem A ([6, Theorem 2.1]).

Assume that $\left(\mathrm{A}_{1}\right)-\left(\mathrm{A}_{5}\right)$ hold, let the functions $H, h$, $k$ satisfy $\left(\mathrm{H}_{1}\right)$ and $\left(\mathrm{H}_{2}\right)$, suppose

$$
\limsup _{t \rightarrow \infty}\left[\lambda C_{1} F(t, r)-\frac{1}{4 \lambda C_{2}} G(t, r)\right]=\infty
$$


holds for every $r \geq t_{0}, C_{1}>0, C_{2}>0$, where

$$
\begin{aligned}
& F(t, r)=\frac{1}{H(t, r)} \int_{r}^{t} H(t, s) k(s) \sum_{j=1}^{l} q_{j}(s) \mathrm{d} s, \\
& G(t, r)=\frac{1}{H(t, r)} \int_{r}^{t} \frac{k(s) h^{2}(t, s)}{H(t, s) \sigma_{j}^{n-2}(s) \sigma_{j}^{\prime}(s)} \mathrm{d} s
\end{aligned}
$$

and $\lambda=1-p$, then every solution of Equation (1) is oscillatory.

Theorem B ([6, Theorem 2.2]).

Assume that $\left(\mathrm{A}_{1}\right)-\left(\mathrm{A}_{5}\right)$ hold, and $H, h, k$ are the same as in Theorem $A$, suppose that

$$
\inf _{s \geq t_{0}}\left\{\liminf _{t \rightarrow \infty} \frac{H(t, s)}{H\left(t, t_{0}\right)}\right\}>0
$$

and

$$
\limsup _{t \rightarrow \infty} G\left(t, t_{0}\right)<\infty
$$

If there exists a function $m \in C\left(\left[t_{0}, \infty\right), R\right)$ such that for all $t \geq T \geq t_{0}$.

$$
\liminf _{t \rightarrow \infty}\left[\lambda C_{1} F(t, T)-\frac{1}{4 \lambda C_{2}} G(t, T)\right] \geq m(T)
$$

and

$$
\limsup _{t \rightarrow \infty} \int_{t_{0}}^{t} \frac{\sigma_{j}^{n-2}(s) \sigma_{j}^{\prime}(s) m_{+}^{2}(s)}{k(s)} \mathrm{d} s=\infty, j=1,2, \cdots, l
$$

where $m_{+}(t)=\max \{m(t), 0\}$, then every solution of Equation (1) is oscillatory.

In Theorem $\mathrm{A}$ and $\mathrm{B}$, function $G(t, r)$ should be $G_{j}(t, r)$, so each of the condition (2), (4), (5) and (6) has as many as $l$ conditions. Meanwhile, the Riccati function $\omega(t)$ is not well-defined and there exist some small errors in the proof of the theorems. The purpose of this paper is further to strengthen oscillation results obtained for Equation (1) by Meng and Xu [6]. In our paper, we redefine the functions $F(t, r), G(t, r), \omega(t)$ and provide some new oscillation criteria for oscillation of Equation (1).

\section{Main Results}

In the sequel, we need the following lemmas:

Lemma 2.1 ([1]).

Let $x(t)$ be a $n$ times differentiable function on $\left[t_{0}, \infty\right)$ of one sign, $x^{(n)}(t) \neq 0$ on $\left[t_{0}, \infty\right)$ which satisfies $x^{(n)}(t) x(t) \leq 0$. Then:

$\left(\mathrm{I}_{1}\right)$ There exists a $t_{1} \geq t_{0}$ such that $x^{(i)}(t), i=1,2, \cdots, n-1$ are of one sign on $\left[t_{1}, \infty\right)$;

$\left(\mathrm{I}_{2}\right)$ There exists a number $h \in\{1,3,5, \cdots, n-1\}$ when $n$ is even, or $h \in\{2,4,6, \cdots, n-1\}$ when $n$ is odd, such that $x(t) x^{(i)}(t)>0$ for $i=0,1, \cdots, h, t \geq t_{1}$; $(-1)^{n+i+1} x(t) x^{(i)}(t)>0$ for $i=h+1, h+2, \cdots, n, \quad t \geq t_{1}$.

Lemma 2.2 ([1]).

If $x(t)$ is as in Lemma 2.1 and $x^{(n-1)}(t) x^{(n)}(t) \leq 0$ for $t \geq t_{0}$, then for every $\lambda(0<\lambda<1)$, there exists a constant $N>0$, such that $|x(\lambda(t))| \geq N t^{n-1}\left|X^{(n-1)}(t)\right|$ for all large $t$.

Lemma 2.3([7]).

Suppose that $x(t)$ is an eventually positive solution of Equation (1), let $z(t)=x(t)+\sum_{i=1}^{m} p_{i}(t) x\left(\tau_{i}(t)\right)$, then there exists a number $t_{1} \geq t_{0}$ such that $z(t)>0$, $z^{\prime}(t)>0, z^{(n-1)}(t)>0$ and $z^{(n)}(t) \leq 0, t \geq t_{1}$.

\section{Theorem 2.1}

Assume that $\left(\mathrm{A}_{1}\right)$ - $\left(\mathrm{A}_{5}\right)$ hold, let the functions $H, h, k$ satisfy $\left(\mathrm{H}_{1}\right)$ and $\left(\mathrm{H}_{2}\right)$, suppose

$$
\limsup _{t \rightarrow \infty}\left[\lambda M F(t, r)-\frac{\beta}{4 \lambda N} G(t, r)\right]=\infty
$$

holds for every $r \geq t_{0}$ and for some $\beta \geq 1$, where

$$
\begin{aligned}
& F(t, r)=\frac{1}{H(t, r)} \int_{r}^{t} H(t, s) k(s) q(s) \mathrm{d} s \\
& G(t, r)=\frac{1}{H(t, r)} \int_{r}^{t} \frac{k(s) h^{2}(t, s)}{H(t, s) \sum_{j=1}^{l} \sigma_{j}^{n-2}(s) \sigma_{j}^{\prime}(s)} \mathrm{d} s
\end{aligned}
$$

and $\lambda=1-p$, then every solution of Equation (1) is oscillatory.

Proof. Suppose to the contrary that $x(t)$ is a nonoscillatory solution of Equation (1) and that $x(t)$ is even- tually positive (when $x(t)$ is eventually negative, the proof is similar).

Let $z(t)$ be defined as in Lemma 2.3, then following the proof of Theorem 2.1 in [6], without loss of generality, assume there exists a $t_{1} \geq t_{0}$ such that

$$
\begin{aligned}
& x(t)>0, z(t)>0, z^{\prime}(t)>0, \\
& z^{(n-1)}(t)>0, z\left(\sigma_{j}(t)\right)>z\left(\lambda \sigma_{j}(t)\right)>0
\end{aligned}
$$

$z^{\prime}\left(\lambda \sigma_{j}(t)\right) \geq N \sigma_{j}^{n-2}(t) z^{(n-1)}(t) \quad$ (by lemma 2.2) and $z^{(n)}(t) \leq-\lambda M \sum_{j=1}^{l} q_{j}(t) z\left(\sigma_{j}(t)\right)$ for all $t \geq t_{1}$.

Let

$$
\omega(t)=k(t) \frac{z^{(n-1)}(t)}{\sum_{j=1}^{l} z\left(\lambda \sigma_{j}(t)\right)} \text { (not as [6]), }
$$

then we have 


$$
\omega^{\prime}(t) \leq-\lambda M k(t) q(t)+\frac{k^{\prime}(t)}{k(t)} \omega(t)-\frac{\lambda N \sum_{j=1}^{l} \sigma_{j}^{n-2}(t) \sigma_{j}^{\prime}(t)}{k(t) \omega^{2}(t)}, \quad t \geq t_{1}, \quad \text { (not as [6]). }
$$

Multiplying the above equation, with $t$ replaced by $t \geq T \geq t_{1}$, for some $\beta \geq 1$, we obtain $s$, by $H(t, s)$ and integrating it from $T$ to $t$, for all

$$
\begin{aligned}
& \lambda M \int_{T}^{t} H(t, s) k(s) q(s) \mathrm{d} s \leq H(t, T) \omega(T)+\int_{T}^{t} h(t, s) \omega(s) \mathrm{d} s-\int_{T}^{t} \lambda N \frac{\sum_{j=1}^{l} \sigma_{j}^{n-2}(s) \sigma_{j}^{\prime}(s)}{k(s)} H(t, s) \omega^{2}(s) \mathrm{d} s \\
& =H(t, T) \omega(T)+\frac{\beta}{4} \int_{T}^{t} \frac{k(s) h^{2}(t, s)}{\lambda N H(t, s) \sum_{j=1}^{l} \sigma_{j}^{n-2}(s) \sigma_{j}^{\prime}(s)} \mathrm{d} s-\frac{\beta}{\beta-1} \int_{T}^{t} \frac{\lambda N H(t, s) \sum_{j=1}^{l} \sigma_{j}^{n-2}(s) \sigma_{j}^{\prime}(s)}{k(s)} \omega^{2}(s) \mathrm{d} s \\
& \left.-\int_{T}^{t}\left\{\frac{\sqrt{\lambda N H(t, s) \sum_{j=1}^{l} \sigma_{j}^{n-2}(s) \sigma_{j}^{\prime}(s)}}{\sqrt{\beta k(s)}} \omega(s)-\frac{\sqrt{\beta k(s)}}{\sqrt{4 \lambda N H(t, s) \sum_{j=1}^{l} \sigma_{j}^{n-2}(s) \sigma_{j}^{\prime}(s)}}\right]^{2}(t, s)\right\} \mathrm{d} s \\
& \leq H(t, T) \omega(T)+\frac{\beta}{4} \int_{T}^{t} \frac{k(s) h^{2}(t, s)}{\lambda N H(t, s) \sum_{j=1}^{l} \sigma_{j}^{n-2}(s) \sigma_{j}^{\prime}(s)} \mathrm{d} s
\end{aligned}
$$

Hence, we have

$$
\lambda M F(t, T)-\frac{\beta}{4 \lambda N} G(t, T) \leq \omega(T)
$$

for all $t \geq T \geq t_{1}$, this gives

$$
\limsup _{t \rightarrow \infty}\left[\lambda M F(t, r)-\frac{\beta}{4 \lambda N} G(t, r)\right]<\infty
$$

which contradicts (7). This completes the proof of the Theorem.

The assumption (7) in Theorem 2.1 can fail, consequently, Theorem 2.1 does not apply. The following results provide some essentially new oscillation criteria for Equation (1).

\section{Theorem 2.2}

Assume that $\left(\mathrm{A}_{1}\right)-\left(\mathrm{A}_{5}\right)$ hold, the functions $H, h, k, F$ and $G$ be the same as in Theorem 2.1, suppose that

$$
\inf _{s \geq t_{0}}\left\{\liminf _{t \rightarrow \infty} \frac{H(t, s)}{H\left(t, t_{0}\right)}\right\}>0
$$

If there exists a function $m \in C\left(\left[t_{0}, \infty\right), R\right)$ such that for all $t \geq T \geq t_{0}$ and for some $\beta>1$,

$$
\limsup _{t \rightarrow \infty}\left[\lambda M F(t, T)-\frac{\beta}{4 \lambda N} G(t, T)\right] \geq m(T)
$$

and

$$
\limsup _{t \rightarrow \infty} \int_{t_{0}}^{t} \frac{\sum_{j=1}^{l} \sigma_{j}^{n-2}(s) \sigma_{j}^{\prime}(s) m_{+}^{2}(s)}{k(s)} \mathrm{d} s=\infty
$$

where $m_{+}(t)=\max \{m(t), 0\}$. Then every solution of Equation (1) is oscillatory.

Proof. Assume to the contrary that (1) is non-oscillatory. Following the proof of Theorem 2.1, without loss of generality, assume for all $t \geq T \geq t_{0}$ and for some $\beta>1$, we obtain

$$
\begin{aligned}
& \lambda M \int_{T}^{t} H(t, s) k(s) q(s) \mathrm{d} s \leq H(t, T) \omega(T) \\
& +\frac{\beta}{4} \int_{T}^{t} \frac{k(s) h^{2}(t, s)}{\lambda N H(t, s) \sum_{j=1}^{l} \sigma_{j}^{n-2}(s) \sigma_{j}^{\prime}(s)} \mathrm{d} s \\
& -\frac{\beta}{\beta-1} \int_{T}^{t} \frac{\lambda N H(t, s) \sum_{j=1}^{l} \sigma_{j}^{n-2}(s) \sigma_{j}^{\prime}(s)}{k(s)} \omega^{2}(s) \mathrm{d} s
\end{aligned}
$$

So, we get

$$
\lambda M F(t, T)-\frac{\beta}{4 \lambda N} G(t, T) \leq \omega(T)-\frac{\beta-1}{\beta} \lambda N B(t, T)
$$

where

$$
B(t, r)=\frac{1}{H(t, r)} \int_{r}^{t} \frac{H(t, s) \sum_{j=1}^{l} \sigma_{j}^{n-2}(s) \sigma_{j}^{\prime}(s)}{k(s)} \omega^{2}(s) \mathrm{d} s,
$$


then

$$
\begin{array}{r}
\limsup _{t \rightarrow \infty}\left[\lambda M F(t, T)-\frac{\beta}{4 \lambda N} G(t, T)\right] \\
\leq \omega(T)-\frac{\beta-1}{\beta} \lambda N \liminf _{t \rightarrow \infty}(t, T)
\end{array}
$$

For all $T \geq t_{0}$ and for any $\beta>1$, by (9) we have

$$
\omega(T) \geq m(T)+\frac{\beta-1}{\beta} \lambda N \liminf _{t \rightarrow \infty} B(t, T)
$$

So

$$
\omega(T) \geq m(T)
$$

and especially

$$
\liminf _{t \rightarrow \infty} B\left(t, t_{0}\right) \leq \frac{\beta}{(\beta-1) \lambda N}\left[\omega\left(t_{0}\right)-m\left(t_{0}\right)\right]<\infty
$$

Now, we claim that

$$
\limsup _{t \rightarrow \infty} \int_{t_{0}}^{t} \frac{\sum_{j=1}^{l} \sigma_{j}^{n-2}(s) \sigma_{j}^{\prime}(s)}{k(s)} \omega^{2}(s) \mathrm{d} s<\infty
$$

Suppose to the contrary that

$$
\limsup _{t \rightarrow \infty} \int_{t_{0}}^{t} \frac{\sum_{j=1}^{l} \sigma_{j}^{n-2}(s) \sigma_{j}^{\prime}(s)}{k(s)} \omega^{2}(s) \mathrm{d} s=\infty
$$

By (8), there is a positive constant $\xi$ satisfying

$$
\inf _{s \geq t_{0}}\left\{\liminf _{t \rightarrow \infty} \frac{H(t, s)}{H\left(t, t_{0}\right)}\right\} \geq \xi>0
$$

Let $\eta$ be any arbitrary positive number, from (14) there exists a $t_{1}>t_{0}$ such that,

$$
\int_{t_{0}}^{t} \frac{\sum_{j=1}^{l} \sigma_{j}^{n-2}(s) \sigma_{j}^{\prime}(s)}{k(s)} \omega^{2}(s) \mathrm{d} s \geq \frac{\eta}{\xi} \quad \text { for all } t>t_{1}
$$

Then, for $t>t_{1}$, we have $B\left(t, t_{0}\right)$

$$
\begin{aligned}
& =\frac{1}{H\left(t, t_{0}\right)} \int_{t_{1}}^{t} H(t, s) d\left\{\int_{t_{0}}^{s} \frac{\sum_{j=1}^{l} \sigma_{j}^{n-2}(v) \sigma_{j}^{\prime}(v)}{k(v)} \omega^{2}(v) \mathrm{d} v\right\} \\
& \geq \frac{1}{H\left(t, t_{0}\right)} \int_{t_{1}}^{t} H(t, s)\left\{\int_{t_{0}}^{s} \frac{\sum_{j=1}^{l} \sigma_{j}^{n-2}(v) \sigma_{j}^{\prime}(v)}{k(v)} \omega^{2}(v) \mathrm{d} v\right\} \mathrm{d} s
\end{aligned}
$$

$\geq \frac{\eta}{\xi} \frac{H\left(t, t_{1}\right)}{H\left(t, t_{0}\right)}$
By (15), there exists a $t_{2} \geq t_{1}$ such that, for all $t>t_{2}, \frac{H\left(t, t_{1}\right)}{H\left(t, t_{0}\right)} \geq \xi$, which implies $B\left(t, t_{0}\right) \geq \eta$ for all $t>t_{2}$. Since $\eta$ is arbitrary, we have

$$
\liminf _{t \rightarrow \infty} B\left(t, t_{0}\right)=\lim _{t \rightarrow \infty} B\left(t, t_{0}\right)=\infty
$$

which contradicts (12), thus (13) holds. Then by (11) and (13) we get

$$
\begin{aligned}
& \limsup _{t \rightarrow \infty} \int_{t_{0}}^{t} \frac{\sum_{j=1}^{l} \sigma_{j}^{n-2}(s) \sigma_{j}^{\prime}(s)}{k(s)} m_{+}^{2}(s) \mathrm{d} s \\
& \leq \limsup _{t \rightarrow \infty} \int_{t_{0}}^{t} \frac{\sum_{j=1}^{l} \sigma_{j}^{n-2}(s) \sigma_{j}^{\prime}(s)}{k(s)} \omega^{2}(s) \mathrm{d} s<\infty,
\end{aligned}
$$

which contradicts (10). This completes the proof.

Remark 1 Let $\beta=1$ in Theorem 2.1, Theorem 2.1 reduces to Theorem A [6]; we obtain the same result in Theorem 2.2 in which we omit the assumption (4) in Theorem B [6]. Therefore, Theorem 2.1 and 2.2 are generalizations and improvements of the results obtained in [6].

Remark 2 With an appropriate choices of the functions $H, h$ and $k$, one can derive a number of oscillation criteria for Equation (1) from our theorems.

Let $k(t) \equiv 1, \alpha>0$ is a constant, $H(t, s)=(t-s)^{\alpha}$, $h(t, s)=-\alpha(t, s)^{\alpha-1} \quad t \geq s \geq t_{0}$, and we have

$$
\lim _{t \rightarrow \infty} \frac{H(t, s)}{H\left(t, t_{0}\right)}=\lim _{t \rightarrow \infty} \frac{(t, s)^{\alpha}}{\left(t, t_{0}\right)^{\alpha}}=1 \quad \text { for any } s \geq t_{0} .
$$

Consequently, let $\alpha=2$, using Theorem 2.2, we have:

Corollary 2.1 Assume that $\left(A_{1}\right)-\left(A_{5}\right)$ and (8) hold, suppose that there exists a function $m \in C\left(\left[t_{0}, \infty\right), R\right)$ such that, for some $\beta>1$,

$$
\begin{aligned}
& \limsup _{t \rightarrow \infty} \frac{1}{t^{2}} \int_{T}^{t}\left[\lambda M(t-s)^{2} q(s)-\frac{\beta}{\lambda N \sum_{j=1}^{l} \sigma_{j}^{n-2}(s) \sigma_{j}^{\prime}(s)}\right] \mathrm{d} s \\
& \geq m(T), \quad t \geq T \geq t_{0}
\end{aligned}
$$

and (10) (with $k(s) \equiv 1$ ) hold. Then every solution of Equation (1) is oscillatory.

Example 1 Let $t \in[4, \infty)$, consider the following second order neutral differential equation

$$
[x(t)+p(t) x(\tau(t))]^{\prime \prime}+q(t) x(\sigma(t))=0
$$


where $p(t) \equiv \frac{1}{2}, q(t)=\max \{2(1+t) \sin t, 0\}, f(x)=x$, $\sigma(t)=\int_{4}^{t} \frac{4}{(1+s)(2+\sin s)} \mathrm{d} s$, in this case $M=1$, Let $N=1, \beta=2$, by direct calculation, we get

$$
\begin{aligned}
& \limsup _{t \rightarrow \infty} \frac{1}{t^{2}} \int_{T}^{t}\left[\lambda M(t-s)^{2} q(s)-\frac{\beta}{\lambda N \sigma^{\prime}(s)}\right] \mathrm{d} s \\
& \geq \lim _{t \rightarrow \infty} \sup \frac{1}{t^{2}} \int_{T}^{t}\left[(t-s)^{2}(1+s) \sin s-(1+s)(2+\sin s)\right] \mathrm{d} s \\
& \stackrel{\Delta}{=} m(T)=\cos T-\sin T+T \cos T-1
\end{aligned}
$$

It is easy to verify that (10) holds, therefore, Equation (17) is oscillatory by Corollary 2.1. However, we can easily find that

$$
\limsup _{t \rightarrow \infty} G\left(t, t_{0}\right)=\limsup _{t \rightarrow \infty} \frac{1}{t^{2}} \int_{t_{0}}^{t}(1+s)(2+\sin s) \mathrm{d} s=\infty
$$

so condition (4) in Theorem B is not satisfied, these show that Theorem B cannot be applied to Equation (17). Obviously our results are superior to the results obtained before.

\section{Acknowledegments}

The authors are very grateful to the referee for his/her valuable suggestions.

\section{References}

[1] R. P. Agarwal, S. R. Grace and D. ORegan, "Oscillation Theory for Differential Equations,” Kluwer Academic, Dordrecht, 2000.

[2] R. P. Agarwal and S. R. Grace, "The Oscillation of Higher Order Differential Equations with Deviating Arguments," Computers \& Mathematics with Applications, Vol. 38, No. 3-4, 1999, pp. 185-199. doi:10.1016/S0898-1221(99)00193-5

[3] Y. Bolat and O. Akin, "Oscillatory Behavior of Higher Order Neutral Type Nonlinear Forced Differential Equation with Oscillating Coefficients," Journal of Mathematical Analysis and Applications, Vol. 290, No. 1, 2004, pp. 302-309. doi:10.1016/j.jmaa.2003.09.062

[4] W. N. Li, "Oscillation of Higher Order Delay Differential Equations of Neutral Type,” The Georgian Mathematical Journal, Vol. 7, No. 2, 2000, pp. 347-353.

[5] Ch. G. Philos, "Oscillation Theorems for Linear Differential Equations of Second Order," Archiv der Mathematik, Vol. 53, No. 5, 1989, p. 483. doi:10.1007/BF01324723

[6] F. Meng and R. Xu, "Kamenev-Type Oscillation Criteria for Even Order Neutral Differential Equations with Deviating Arguments," Applied Mathematics and Computation, Vol. 190, No. 2, 2007, pp. 1402-1408. doi:10.1016/j.amc.2007.02.017

[7] Yu. V. Rogovchenko and F. Tuncay, “Oscillation Criteria For Second-Order Nonlinear Differential Equations with Damping," Nonlinear Analysis: Theory, Methods \& Applications, Vol. 69, No. 1, 2008, pp. 208-221. doi:10.1016/j.na.2007.05.012 